\title{
GALIUS: an ultrafast imaging spectrograph for the study of lightning
}

\author{
María Passas-Varo ${ }^{1,}{ }^{*}$, Justo Sánchez ${ }^{1}$, Thi Ny KieU ${ }^{1}$, Ernesto Sánchez-Blanco ${ }^{2}$, And \\ FRANCISCO J. GORDILLO-VÁZQUEZ ${ }^{1, * *}$ \\ ${ }^{1}$ Instituto de Astrofísica de Andalucía (IAA), CSIC, PO Box 3004, 18080 Granada, Spain \\ ${ }^{2}$ Optical Development, Consultant, Avenida Pablo Iglesias 7 Local 5, 41008 Sevilla, Spain \\ *Corresponding author: passasv@iaa.es \\ *e-mail: vazquez@iaa.es
}

Compiled November 14, 2019

\begin{abstract}
We present the main parameters, design features and optical characterization of the GrAnada LIghtning Ultrafast Spectrograph (GALIUS): a portable, ground-based spectrographic system intended for the analysis of the spectroscopic signature of lightning. It has been designed to measure the spectra of the light emitted from natural and triggered lightning and artificial electrostatic discharges at recording speeds up to 2.1 Mfps. It includes a set of four interchangeable grisms covering different spectral ranges (from 375 $\mathrm{nm}$ to $854.5 \mathrm{~nm}$ ) with spectral resolutions from 0.29 to $0.76 \mathrm{~nm}$. A set of ten collector lenses allows to record the spectrum of electrostatic discharges and lightning in different scenarios. (๑) 2019 Optical Society of America
\end{abstract}

http://dx.doi.org/10.1364/ao.XX.XXXXXX

\section{INTRODUCTION}

Lightning spectroscopy is a matter of interest to the atmospheric research community since midnineteenth century. Specially nowadays, when the progress of technology makes possible the improvement of both temporal and spatial resolution (XR) of the measurement of lightning spectrum thanks to the incipient development of CCD and CMOS ultra-high speed cameras and volume phase holografic (VPH, hereinafter) diffraction gratings.

The fastest spectrograph to date designed to record lightning spectrum was developed by the Department of Atmospheric Science of the University of Alabama in Huntsville, with a maximum recording speed of $1 \mathrm{Mfps}[1,2]$. They recorded the first high-speed slit less spectra of triggered lightning at $660000 \mathrm{fps}$, distinguishing its different phases and identifying neutral, singly and doubly ionized nitrogen, oxygen and some neutral argon lines, with a lack of any traces of molecular emissions [1].

Higher speed measurements would give an idea of the initial temporal stages of, for instance, lightning leaders or how high can become the temperature in lightning return stroke channels. In this sense, we have designed, developed and calibrated the GrAnada LIghtning Ultrafast Spectrograph (GALIUS, hereinafter), the first portable ultra high speed spectrograph designed to analyze key properties such as electron density and gas temperature of lightning and high voltage (HV) electrostatic discharges up to $2.1 \mathrm{Mfps}$.
2. GALIUS

A. Experimental device

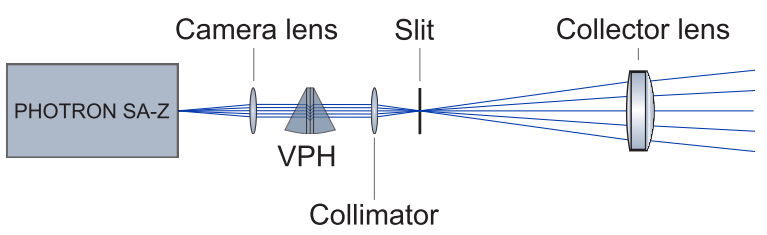

Fig. 1. Optical scheme of the GALIUS CMOS imaging spectrograph.

Figure 1 shows the optical scheme of GALIUS. A total of 22 configurations can be set up, combining ten interchangeable collector lenses with four interchangeable grisms. The collimator characteristics also vary, depending on the spectral range we want to measure (see table 1 ). Table 2 shows the characteristics of the four different VPHs: R1 to measure the UV spectral range and $\mathrm{R} 2, \mathrm{R} 3$ and $\mathrm{R} 4$ to measure the visible-NIR spectral range. Let's call the $U V$ setup to the configuration resulting from combining R1 with collector lenses A, B, C or D. And let's call the Visible-NIR setup to the possible configuration resulting from combining R2, R3 or R4 with collector lenses E, F, G, H, I or J.

The optical scheme of GALIUS, following the light path, consists of a collector lens (see table 3), an optional slit of $50 \mu \mathrm{m} \mathrm{x}$ 
Table 1. GALIUS interchangeable collimators

\begin{tabular}{lcc}
\hline Spectral range & Focal length & F\# \\
UV & $105 \mathrm{~mm}$ & 4.5 \\
Visible-NIR & $50 \mathrm{~mm}$ & 1.2 \\
\hline
\end{tabular}

$3 \mathrm{~mm}$, a collimator (see table 1), a grism (see table 2), a camera lens of $50 \mathrm{~mm}$ focal length and F\#1.2 and a PHOTRON SA-Z CMOS ultrahigh speed camera. Optical rails and holders set all the components inside a portable housing (see figure 2). In the UV scenario, we place a $345 \mathrm{~nm}$ high pass filter between the collimator and the VPH to avoid second order spectra between 200 and $400 \mathrm{~nm}$ in the spectral response of a commercial spectrograph that we used to calculate GALIUS instrument function. GALIUS also includes a photometer that triggers the Photron SA-Z camera when a threshold of photons is detected, and a WATEC WAT-902H2 ultimate with a 3005VX4 sensor wide-field camera, to discern the origin of the recorded spectra.

\section{A.1. Housing}

GALIUS is housed in a portable aluminum box of $1100 \mathrm{~mm} \times 380$ $\mathrm{mm} \times 230 \mathrm{~mm}$, which can be bent in elevation from $0^{\circ}$ until $50^{\circ}$ thanks to a linear actuator and a telescopic mechanism attached on its fixed base (see figure 2).

\section{A.2. Grisms}

We have designed four interchangeable grisms. They consist of an embedded VPH transmission grating between two prisms so the path of the light keeps straight. Table 2 summarizes the specifications of the grisms R1 (UV), R2 (Visible-NIR), R3 (Visible-NIR) and R4 (Visible-NIR).

\section{A.3. Sensor}

The ultra-high speed Photron FASTCAM SA-Z includes a highly light sensitive image sensor (monochrome ISO 50,000) of 1024 x $102420 \mu \mathrm{m}$ square pixels, with 12-bit ADC pixel depth. It provides frame rates up to 2.1 million frames per second (fps) at reduced image resolution. Table 4 summarizes the maximum resolution of the Photron FASTCAM SA-Z sensor, depending on the frame rate.

\section{A.4. Photometer and triggering system}

The photometer is based on a fast photodiode working under photoconductive mode. It amplifies the optical signal and launches a Transistor - Transistor Logic (TTL) synchronism pulse that triggers the Photron SA-Z camera whenever a threshold in a number of photons is reached. The photometer can either launch a positive or negative TTL pulse with a typical delay of $20 \mathrm{~ns}$. The photometer is very useful to synchronize the Photron SA-Z recording with the initial stage of a spark or lightning and to avoid collapsing the internal buffer of the camera with empty images.

\section{B. Spectrograph calibration}

In order to calibrate GALIUS we followed the steps previously described for calibrating the GRAnada Sprite Spectrograph and Polarimeter (GRASSP) [3] presently in operation for high spectral resolution TLE spectroscopy [4-7]. The detailed steps followed to calibrate GALIUS are: (1) a distortion correction, to straighten the curved raw spectrum; (2) a wavelength calibration, to assign an absolute wavelength to each pixel of the CMOS;
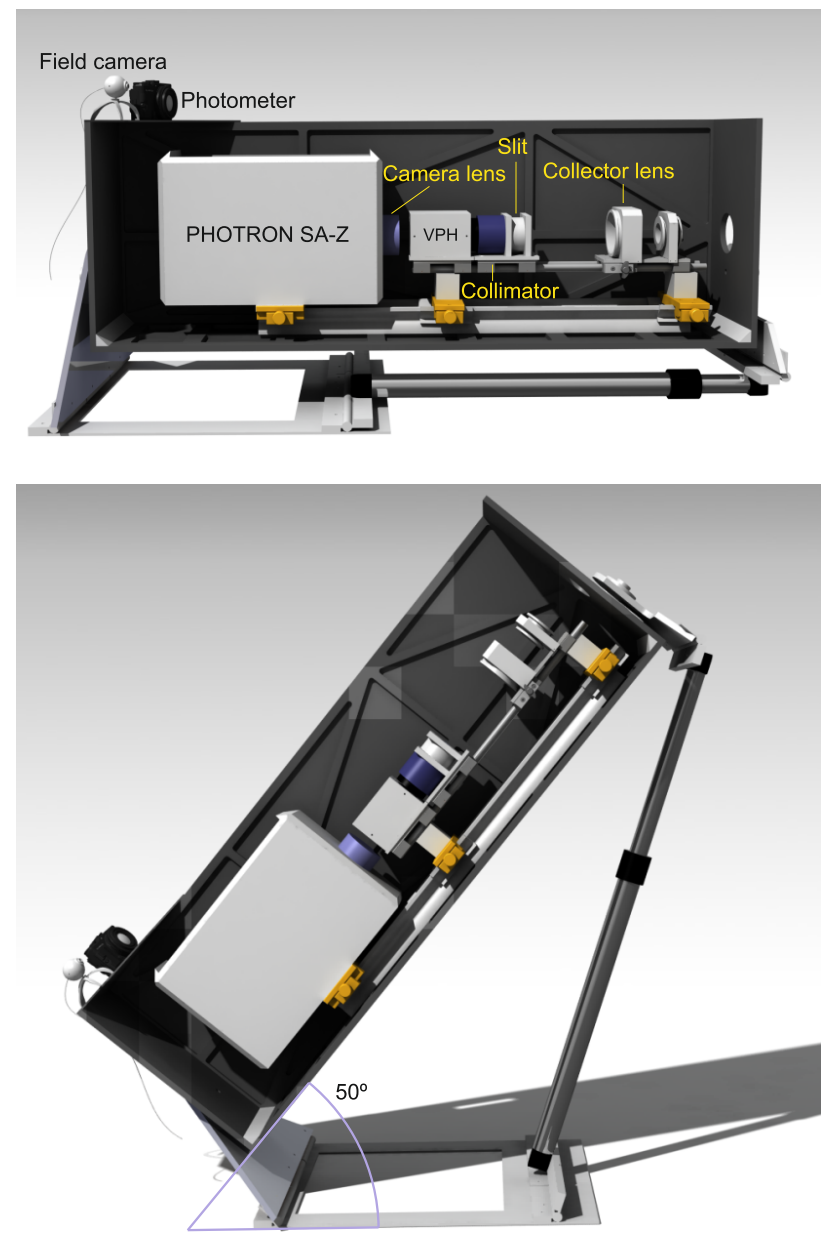

Fig. 2. GALIUS housing

and (3) a flux calibration, to provide the spectral sensitivity of the spectrograph. Distortion correction and wavelength calibration are done whenever a spectrum is recorded; the 22 different instrument functions (one per each configuration of GALIUS) are calculated once a year.

\section{B.1. Distortion Correction and Wavelength Calibration}

We have implemented a software in Python to correct the curvature of the spectrum due to the aberration introduced by the optical elements of GALIUS. We recorded the spectrum of deuterium (for the UV setup) and neon and argon (for the Visible-NIR setup) spectral lamps. Then, we trimmed them so all spectral lines appeared in every row of the spectrum. Then, we identified the corresponding wavelengths by comparing the distances between the spectral lines to the gaps that deuterium, neon and argon spectra show. We measured the deuterium spectrum with a commercial Jobin Yvon Horiba FHR1000 spectrograph and we obtained the $\mathrm{Ne}$ and Ar spectral lines from [8]. Finally, to straighten the spectrum, we assigned the central pixel of each spectral line to a single wavelength for every row of the spectral image. This software generates a calibration matrix that will be used to correct the distorted spectra recorded with GALIUS, associating every pixel coordinate of the CMOS to an absolute wavelength. This calibration matrix is calculated every time we 
Table 2. GALIUS configurations

\begin{tabular}{ccccc}
\hline Grism & Lines/mm & Spectral resolution & Spectral range & Collector lenses \\
R1 & 2086 & $0.2945 \mathrm{~nm}$ & $380 \mathrm{~nm}-450 \mathrm{~nm}$ & A, B, C, D \\
R2 & 1015 & $0.7530 \mathrm{~nm}$ & $464 \mathrm{~nm}-794 \mathrm{~nm}$ & E, F, G, H, I, J \\
R3 & 1855 & $0.3831 \mathrm{~nm}$ & $587.7 \mathrm{~nm}-737.8 \mathrm{~nm}$ & E, F, G, H, I, J \\
R4 & 1727 & $0.3456 \mathrm{~nm}$ & $706.7 \mathrm{~nm}-854.5 \mathrm{~nm}$ & E, F, G, H, I, J \\
\hline
\end{tabular}

perform a new set of measurements.

Table 3. GALIUS interchangeable collector lenses

\begin{tabular}{cccc}
\hline Lens & Focal length & F\# & Spectral range \\
A & $25 \mathrm{~mm}$ & 1 & $250-425 \mathrm{~nm}$ \\
$\mathrm{~B}$ & $30 \mathrm{~mm}$ & 1.50 & $250-425 \mathrm{~nm}$ \\
$\mathrm{C}$ & $38 \mathrm{~mm}$ & 1.52 & $250-425 \mathrm{~nm}$ \\
$\mathrm{D}$ & $50 \mathrm{~mm}$ & 1.50 & $250-425 \mathrm{~nm}$ \\
$\mathrm{E}$ & $25 \mathrm{~mm}$ & 1.67 & $400-1000 \mathrm{~nm}$ \\
$\mathrm{~F}$ & $30 \mathrm{~mm}$ & 1.50 & $400-1000 \mathrm{~nm}$ \\
$\mathrm{G}$ & $40 \mathrm{~mm}$ & 1.60 & $400-1000 \mathrm{~nm}$ \\
$\mathrm{H}$ & $50 \mathrm{~mm}$ & 1.67 & $400-1000 \mathrm{~nm}$ \\
$\mathrm{I}$ & $60 \mathrm{~mm}$ & 1.50 & $400-1000 \mathrm{~nm}$ \\
$\mathrm{~J}$ & $200 \mathrm{~mm}$ & 2.70 & $400-1000 \mathrm{~nm}$ \\
\hline
\end{tabular}

\section{B.2. Flux Calibration}

We measured the spectral response of the 22 different GALIUS setups.

For the UV setup, we measured with GALIUS the spectral radiance of a deuterium lamp (see figure 3) directly from the bulb at 50 frames per second located at $24.5 \mathrm{~cm}$ from the collector lens, measuring in 3 different regions of the beam, and combining these measurements by terms of the median value of images. We repeated the setup and measured the spectral radiance of the same deuterium lamp with a calibrated Jobin Yvon HORIBA FHR1000 spectrometer. In this case we located the optic fiber over 9 different regions of the deuterium beam with a micrometric regulation screw, and we combined these measurements as their median value. Then we proceeded as in [9]. It is worth highlighting that we could not use an integrating sphere since the radiance from the lamp is very weak for the UV spectral range, and the system sensitivity is also very low at these wavelengths. From these calculations we got 4 different instrumental functions.

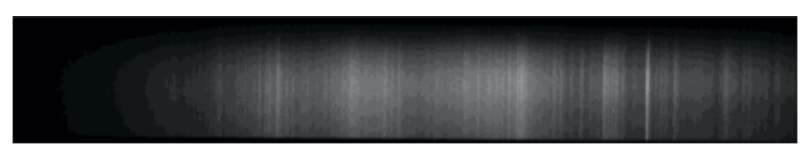

Fig. 3. Spectral image of a deuterium lamp recorded with GALIUS for grism R1 using the collector lens D (50 $\mathrm{mm}$ focal length).

For the Visible-NIR setups, we repeated the steps defined in [3]. We recorded the spectral radiance of a halogen tungsten spectral lamp (QTH) at 50 frames per second located at $60 \mathrm{~cm}$ from the collector lens, and repeated these measurements with the calibrated Jobin Yvon HORIBA FHR1000 spectrometer using the same calibration setup. From these calculations we got 18 different instrumental functions.

Equation 1 provides the instrument function of GALIUS for row $i$ of the CMOS, according to the steps defined in [9]:

$$
\operatorname{IF}(\lambda, i)=\frac{S_{F H R 1000}(\lambda)}{S_{\text {GALIUS }}(\lambda, i)}
$$

As the spectral resolutions of the FHR1000 and GALIUS are different, we could not divide them directly to calculate the spectral sensitivity. To correct this issue we convolved both responses with a gaussian curve so the resulting signals had the same spectral resolution. Figure 4 shows the spectral sensitivity of row number 16 for grating R1 with a collector lens of $200 \mathrm{~mm}$ focal length. 


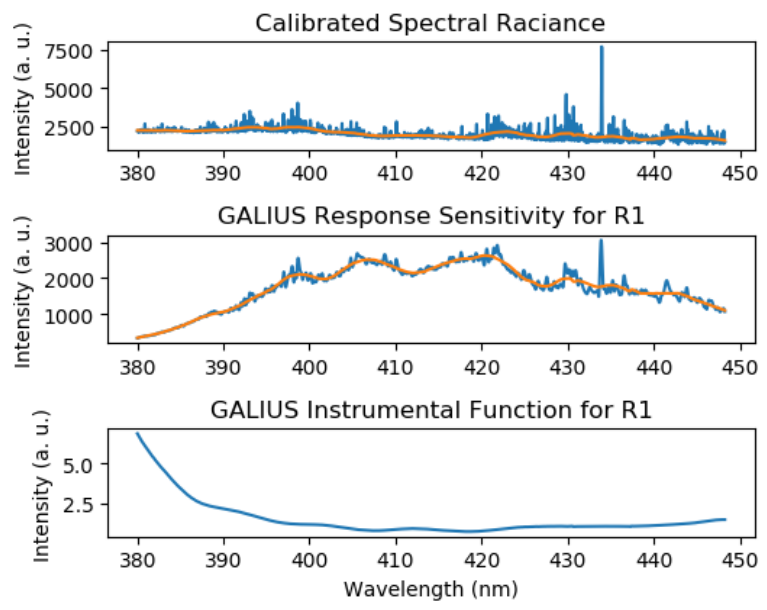

Fig. 4. Spectral sensitivity of GALIUS for grism R1 using the collector lens D (50 mm focal length) for CMOS row number 16.

\section{Spectral resolution}

We have estimated the spectral resolution of GALIUS by measuring the full width at half-maximum (FWHM) of different spectral emission lines for each VPH. We also estimated the mean resolving power of the spectrograph as $R=\lambda / \delta \lambda$, with $\delta \lambda$ as the FWHM of a certain spectral emission line. The spectral dispersion (D) and FWHM and R mean values are shown in table 5. The current resolution element is contained in 2.17 to 2.6 pixels average (it changes across field, due to anamorphics and aberrations), which is very close to the Nyquist limit (2 to 2.4 pixels).

Table 5. GALIUS Spectral parameters

\begin{tabular}{cccc}
\hline VPH & D (nm/px) & FWHM (nm) & R \\
R1 & 0.1354 & 0.2945 & 1409.17 \\
R2 & 0.3306 & 0.7530 & 835.36 \\
R3 & 0.1483 & 0.3831 & 1729.97 \\
R4 & 0.1481 & 0.3456 & 2258.68 \\
\hline
\end{tabular}

\section{Spatial dispersion}

We measured the spatial dispersion (XD) of the GALIUS spectrograph for settings F, G, H and J by measuring the spectrum of the Sun reflected on a full Moon, which subtends about $32 \pm 3 \mathrm{~min}$ of arc from Earth. The number of pixels of this spectrum on the CMOS vary in the spatial dimension depending on the collector lens, being linear the ratio between this number of pixels and the collector lens focal length. We were unable to measure the spectrum the Sun reflected on the Moon with the UV setup due to the weakness of the spectrum at these wavelengths, so we estimated the spatial dispersion assuming linearity.

According to Nyquist criterion, the spatial resolution (XR) is three times the spectral dispersion. Table 6 shows the spatial dispersion (second column) and spatial resolution (third column) for each collector lens (first column).

\section{E. Stability}

We have checked the stability of GALIUS in our laboratory for every VPH by recording the spectrum of the calibration lamps within a time interval of seven hours, and we did not detect any drift in the wavelength. However, we will calibrate in wavelength at the beginning and at the end of a working session to check the stability of the system due to movements during the observation night.

Table 6. GALIUS Spatial parameters

\begin{tabular}{ccc}
\hline Lens & XD (arc sec/px) & XR (arc min) \\
A & $279.91 \pm 26.24$ & $14.00 \pm 1.31$ \\
B & $236.84 \pm 22.205$ & $11.84 \pm 1.11$ \\
C & $190.06 \pm 17.82$ & $9.50 \pm 0.89$ \\
D & $146.62 \pm 13.75$ & $7.33 \pm 0.69$ \\
E & $133.29 \pm 12.50$ & $6.66 \pm 0.62$ \\
F & $112.78 \pm 10.57$ & $5.64 \pm 0.53$ \\
G & $86.25 \pm 8.09$ & $4.31 \pm 0.4$ \\
H & $69.82 \pm 6.55$ & $3.49 \pm 0.33$ \\
I & $58.65 \pm 5.50$ & $2.93 \pm 0.27$ \\
J & $18.10 \pm 1.70$ & $0.91 \pm 0.08$ \\
\hline
\end{tabular}

\section{F. Spectrograph reliability}

In figure 5 we compare two sparks spectra. The blue one is the calibrated spectrum of the light emitted by a $5 \mathrm{~cm}$ spark generated with a Whimshurst machine. It was recorded in our laboratory at $84 \mathrm{kfps}$ and $10 \mu \mathrm{s}$ exposure time with GALIUS setting of grism R1 combined with lens D. The orange graph corresponds to the calibrated spectrum of the light emitted by a spark produced by a Van der Graaff generator. It was recorded at $100 \mathrm{kfps}$ and approximately $10 \mu \mathrm{s}$ exposure time with a VPHVIS spectrograph with $0.75 \mathrm{~nm}$ spectral resolution and a grating of 1257 lines / mm [10].

Since we are comparing the spectra of two different electrostatic discharges recorded both with two different spectrographs at two different spectral and temporal resolutions, these spectra are, hence, different. Nevertheless, both spectra are coincident in peaks, and their background level seems to follow the same slope, so this comparison confirms the reliability of the wavelength and flux calibration of GALIUS between 380 and 450 nm.

We have confirmed the same reliability for grisms R2, R3 and R4.

\section{CONCLUSION}

In this paper we have described the design and development as well as the complete optical characterization of GALIUS, a portable, ground-based ultra fast spectrographic system for the analysis of the spectroscopic signature of lightning and artificial electrostatic discharges at recording speeds up to $2.1 \mathrm{Mfps}$ with spectral resolutions varying from $0.29 \mathrm{~nm}$ to $0.76 \mathrm{~nm}$. GALIUS would allow us to remotely estimate physical parameters of the lightning channel, such as the electron density or the gas temperature, among others. It is worth highlighting that GALIUS is 


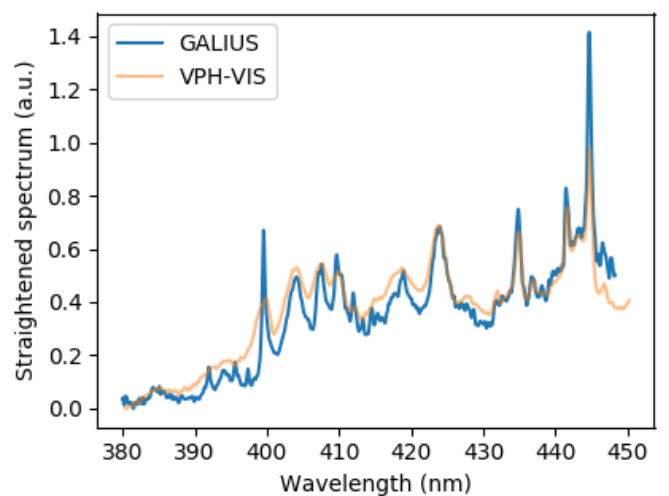

Fig. 5. Spectrum of a spark produced by a Whimshurst machine measured with GALIUS (blue). Spectrum of a spark produced by a small Van der Graaf generator measured with a VPH-VIS spectrograph (orange) [10].

currently the fastest spectrograph intended to analyze lightning spectra, improving the temporal resolution of former lightning slit spectrographs by more than 3 times $[1,2,10]$.

\section{FUNDING}

This work was supported by the Spanish Ministry of Science and Innovation, MINECO, under project ESP2017-86263-C4-4-R and the FEDER program. Additionally, this work has received funding from the European Union Horizon 2020 research and innovation programme under the Marie Sklodowska-Curie grant agreement SAINT 722337. Authors M.P.V, J.S., T.N.K and F.J.G.V. acknowledge financial support from the State Agency for Research of the Spanish MCIU through the "Center of Excellence Severo Ochoa" award for the Instituto de Astrofísica de Andalucía (SEV-2017-0709).

\section{ACKNOWLEDGMENTS}

T. N. K. acknowledges a PhD contract through the project SAINT 722337. Authors gratefully acknowledge Dr. Isabel Tanarro for her invaluable assistance in measuring the calibrated lamps spectra with a Jobin Yvon Horiba FHR1000 spectrometer under her supervision in the Instituto de Estructura de la Materia (IEM), CSIC, Madrid, Spain.

\section{DISCLOSURES}

The authors declare that there are no conflicts of interest related to this article.

\section{REFERENCES}

1. T. D. Walker and H. J. Christian "Triggered lightning spectroscopy: Part I qualitative analysis" J. Geophys. Res. Atmospheres 122, 8000-8011 (2017).

2. T. D. Walker and H. J. Christian "Triggered lightning spectroscopy: Part II quantitative analysis" J. Geophys. Res. Atmospheres 124, 39303942 (2019).

3. M. Passas, J. Sánchez, E. Sánchez-Blanco, A. Luque and F. J. GordilloVázquez "GRASSP: a spectrograph for the study of transient luminous events" Appl. Opt. 55, 23, 6436-6442 (2016).

4. F. C. Parra-Rojas, M. Passas, E. Carrasco, A. Luque, I. Tanarro, M. Simek and F. J. Gordillo-Vázquez "Spectroscopic diagnostics of laboratory air plasmas as a benchmark for spectral rotational (gas) temperature determination in TLEs" J. Geophys. Res. Space Phys. 118 4649-4661 (2013).

5. M. Passas, J. Sánchez, A. Luque and F. J. Gordillo-Vázquez "Transient Upper Atmospheric Plasmas: Sprites and Halos" IEEE Transactions on Plasma Sci. 42 2664-2665 (2014).

6. M. Passas, J. M. Madiedo and F. J. Gordillo-Vázquez "High resolution spectroscopy of an Orionid meteor from 700 to 800 nm" Icarus. 266 134-141 (2016).

7. F. J. Gordillo-Vázquez, M. Passas, A. Luque, J. Sánchez, O. van der Velde and J. Montanyá "High Spectral Resolution Spectroscopy of Sprites: A Natural Probe of the Mesosphere" J. Geophys. Res. Atmospheres 123 2336-2346 (2018).

8. A. Kramida, Yu. Ralchenko, J. Reader and NIST ASD Team "NIST Atomic Spectra Database (ver. 5.2), [Online]. Available: http://physics.nist.gov/asd" Natl. Inst. Standards Technol. Gaithersburg, MD. (2015).

9. U. Fantz "Basics of plasma spectroscopy" Plasma Sources Sci. Technol. 15, 137-147 (2006).

10. T. D. Walker "A 21st Century Investigation of the Lightning Spectrum" PhD Thesis Diss. Univ. Ala. Huntsville (2015). 